J. DIFFERENTIAL GEOMETRY

51 (1999) 13-33

\title{
ON A LINEARITY PROBLEM FOR PROPER HOLOMORPHIC MAPS BETWEEN BALLS IN COMPLEX SPACES OF DIFFERENT DIMENSIONS
}

\author{
XIAOJUN HUANG
}

\section{Introduction}

In an important development of several complex variables, Poincaré [26] discovered that any biholomorphic map between two open pieces of the unit sphere in $\mathbf{C}^{2}$ is the restriction of a certain automorphism of $\mathbf{B}_{2}$, the unit two-ball in $\mathbf{C}^{2}$. This phenomenon fails obviously in one complex variable and reveals a strong rigidity property of holomorphic mappings in several variables. Later, Tanaka, etc (see [8], [28]) extended this result to any dimensional case. Alexander, in his famous papers [1], [2], further proved that any proper holomorphic selfmapping of the ball in $\mathbf{C}^{n}(n>1)$ is an automorphism, thus finishing off a line of research towards the understanding of proper holomorphic mappings between balls in the same complex space. In 1978, using the Cartan-Chern-Moser [8] theory, Webster [31] took up again the problem of considering a proper holomorphic mapping $f$ from the unit $n$-ball $\mathbf{B}_{n}=\left\{z \in \mathbf{C}^{n}:|z|<1\right\}$ into the unit $(n+1)-$ ball $\mathbf{B}_{n+1} \subset \mathbf{C}^{n+1}$ and showed that $f$ is a totally geodesic embedding when $f$ is $C^{3}$-smooth up to the boundary and when $n>2$. Here, we recall that a proper holomorphic map from $\mathbf{B}_{n}$ into $\mathbf{B}_{N}$ is called a totally geodesic embedding (or a linear embedding) if there exist automorphisms $\sigma \in \operatorname{Aut}\left(\mathbf{B}_{n}\right)$ and $\tau \in \operatorname{Aut}\left(\mathbf{B}_{N}\right)$ such that $\tau \circ f \circ \sigma=(\mathrm{id}, 0)$. In a subsequent paper,

Received December 18, 1997, and, in revised form, April 23, 1998. The author was supported in part by NSF DMS-9500881 and an NSF postdoctoral fellowship. 
Faran [14] classified mappings from $\mathbf{B}_{2}$ into $\mathbf{B}_{3}$, that are three times continuously differentiable up to the boundary. In another work [9], Cima-Suffridge studied certain reflection principle for CR mappings between hypersurfaces with codimension one and established the results of Webster and Faran for mappings which are only twice continuously differentiable up to the boundary. In the same paper, they conjectured that any proper holomorphic mapping from $\mathbf{B}_{n}$ into $\mathbf{B}_{N}(n>1)$, which is $C^{2}$-smooth up to the boundary, must be a totally geodesic embedding when $N<2 n-1$. Notice that there are many proper polynomial mappings from $\mathbf{B}_{n}$ into $\mathbf{B}_{2 n-1}$ which are not totally geodesic (see [7], in particular, the book by D'Angelo [13], where many related classifications are presented). Indeed, the well-known (non-linear) Whitney map [10] $f=\left(z_{1}^{2}, z_{1} z_{2}, \cdots, z_{1} z_{n}, z_{2}, \cdots, z_{n}\right)$ properly sends $\mathbf{B}_{n}$ into $\mathbf{B}_{2 n-1}$. In [15], Faran verified this conjecture under the assumption that the map extends holomorphically up to the boundary.

Later, it was shown in the deep work of Forstneric and Cima-Suffridge [17], [18], [10] (see in particular, the recent work in [18]), that any proper holomorphic mapping from $\mathbf{B}_{n}$ into $\mathbf{B}_{N}$, that is $C^{N-n+1}$-regular up to the boundary, extends holomorphically and rationally across the closure of the ball for any $N \geq n \geq 2$. This together with the work of Faran gives a solution to Cima-Suffridge's problem for mappings which are $(N-n)+1(($ codimension $)+1)$ smooth up to the boundary. Meanwhile, in a different direction, the discovery of inner functions over the ball reveals that there are many proper holomorphic mappings from $\mathbf{B}_{n}$ into $\mathbf{B}_{n+1}$ which are continuous up to $\overline{\mathbf{B}}_{n}$ but not linear (see [20], [22], [24], and [27]. In particular, see [19] for a survey on the related topics). This opens up a very interesting but also difficult subject to dig out the minimal boundary regularity for mappings between balls with which the linearity and the reflection principle hold. In particular, it has been an open question for years to obtain results in which the required regularity is independent of the codimension ([9], [6], [19]).

In this paper, we will focus on the linearity portion of the above mentioned problem. We will prove the following theorem, which was first conjectured to be true by Cima-Suffridge in 1983 [9] (see also the question asked in [6] and [18]):

Theorem A. Let $M_{1}$ and $M_{2}$ be two connected open pieces of the boundaries of $\mathbf{B}_{n} \subset \mathbf{C}^{n}$ and $\mathbf{B}_{N} \subset \mathbf{C}^{N}$, respectively. Let $f$ be a nonconstant twice continuously differentiable $C R$ mapping from $M_{1}$ into $M_{2}$. Suppose that $n>1, N<2 n-1$. Then $f$ is the restriction of a 
certain totally geodesic embedding from $\mathbf{B}_{n}$ into $\mathbf{B}_{N}$. More precisely, there exist an automorphism $\sigma \in \operatorname{Aut}\left(\mathbf{B}_{n}\right)$ and an automorphism $\tau \in$ $\operatorname{Aut}\left(\mathbf{B}_{N}\right)$ such that $\tau \circ f \circ \sigma\left(z_{1}, \cdots, z_{n}\right) \equiv\left(z_{1}, \cdots, z_{n}, 0, \cdots, 0\right)$. In particular, $f$ is real analytic over $M_{1}$.

Corollary B. Let $f$ be a proper holomorphic mapping from $\mathbf{B}_{n}$ into $\mathbf{B}_{N}$, that is twice continuously differentiable up to the boundary. Suppose that $n>1, N<2 n-1$. Then there exist $\sigma \in \operatorname{Aut}\left(\mathbf{B}_{n}\right)$ and $\tau \in \operatorname{Aut}\left(\mathbf{B}_{N}\right)$ such that $\tau \circ f \circ \sigma\left(z_{1}, \cdots, z_{n}\right) \equiv\left(z_{1}, \cdots, z_{n}, 0, \cdots, 0\right)$.

Our approach to the proof of Theorem $A$ is different from the existing ones for the study of mappings between real analytic hypersurfaces in different complex spaces ([31], [14]-[16], [9]-[10], [17]-[19], [21], [5], etc). Namely, we do not use the differential-geometric part of the CartanChern-Moser theory, and we do not take too many times differentiation by $\mathrm{CR}$ vector fields along the sphere, neither. This enables us to deal with maps with only $C^{2}$-smooth regularity. Indeed, our consideration uses only the lower order Moser formal theory and the large automorphism groups on the balls. This method seems also to be very useful for studying other related problems for mappings between balls (such as rationality and degree estimate problems, etc).

Another implication of our theorem is the analyticity result of $f$. Regularity of CR mappings between hypersurfaces in the same complex space has attracted considerable attentions in recent years and many results have been obtained (see [3] and the survey papers [6], [19]). However, the understanding of regularity of CR mappings between hypersurfaces in different complex spaces is still rather poor (see [16], [11], [18], [21], [5]). We mention that in all previous work, it was always essential to assume that the map under study is at least (codimension +1 )times continuously differentiable to boost-up higher regularity. It seems that the theorem of this paper also gives the first reflection principle for maps whose initial regularity is independent of the codimension.

Finally, we would like to mention a different but closely related interesting problem ([12], [32]), which stems from problems establishing the Mostow super-rigidity theorem to complex hyperbolic space forms (see [12] for a solution in the small codimensional case and [32] for certain related applications to the study of dynamics): Let $f$ be a proper holomorphic embedding from $\mathbf{B}_{n}$ into $\mathbf{B}_{N}$ such that $f\left(\mathbf{B}_{n}\right)$ is stabilized by a certain discrete subgroup $\Gamma$ of $\operatorname{Aut}\left(\mathbf{B}_{N}\right)(N>n>1)$ with $f\left(\mathbf{B}_{n}\right) / \Gamma$ compact. Is $f$ then a totally geodesic embedding?

Acknowledgement. The author thanks J. D'Angelo, Shanyu Ji, 
S. Krantz, Song-Ying Li, Steve S. T. Yau and Chengbo Yue for their interest to the present work. He appreciates greatly S. Baouendi and L. Rothschild for their very careful reading of the paper and many helpful comments. Also, the author would like to thank S. Webster, from whom he first learnt the beauty of the Moser formal theory.

\section{Basic set-ups, a normalization property and a Preliminary Lemma}

We let $M_{1} \subset \partial \mathbf{B}_{n}$ and $M_{2} \subset \partial \mathbf{B}_{N}$ be two connected open pieces of the unit spheres in $\mathbf{C}^{n}$ and $\mathbf{C}^{N}$, respectively. After a linear fractional transformation, we can assume that $M_{1}, M_{2}$ contain the origin and are defined, respectively, by the following equations:

$$
\operatorname{Im} w=\sum_{j=1}^{n-1}\left|z_{j}\right|^{2},(z, w) \in M_{1} ; \operatorname{Im} w^{*}=\sum_{j=1}^{N-1}\left|z_{j}^{*}\right|^{2},\left(z^{*}, w^{*}\right) \in M_{2} .
$$

Write $L_{j}=2 i \overline{z_{j}} \frac{\partial}{\partial w}+\frac{\partial}{\partial z_{j}}$ for $j=1, \cdots, n-1$. Then $\left\{L_{1}, \cdots, L_{n-1}\right\}$ forms a global basis for the complex tangent bundle $\mathrm{T}^{(1,0)} M_{1}$ of $M_{1}$. Let

$$
F=(f, \phi, g)=\left(f_{1}, \cdots, f_{n-1}, \phi_{1}, \cdots, \phi_{N-n}, g\right)
$$

be a non-constant $C^{2}$-smooth CR map from $M_{1}$ into $M_{2}$ with $F(0)=$ 0 . We recall that the $\mathrm{CR}$ assumption of $F$ indicates that $\overline{L_{j}} F \equiv 0$ for each $j$. By the Lewy extension theorem, it is well-known that $F$ extends holomorphically to a certain pseudoconvex side of $M_{1}$ denoted by $\Omega$. Here, we can also assume that $\Omega$ is filled in by holomorphic disks attached to $M_{1}$. Now, applying the maximum principle and the Hopf lemma to the subharmonic function: $-\operatorname{Im} g+\sum_{j=1}^{n-1}\left|f_{j}\right|^{2}+\sum_{j=1}^{N-n}\left|\phi_{j}\right|^{2}$ over $\Omega$, we conclude that $F(\Omega) \subset \mathbf{H}_{N}=\left\{\left(z^{*}, w^{*}\right): \operatorname{Im}\left(w^{*}\right)>\left|z^{*}\right|^{2}\right\}$ and $\left.\frac{\partial(\operatorname{Im} g)}{\partial(\operatorname{Im} w)}\right|_{0}=\lambda>0$. Since $\left.\frac{\partial(\operatorname{Im} g)}{\partial(\operatorname{Re} w)}\right|_{0}=0,\left.\frac{\partial g}{\partial w}\right|_{0}=\lambda$.

Next, we notice that the assumption $F\left(M_{1}\right) \subset M_{2}$ gives the following non-linear functional equation with respect to $F$ :

$$
\frac{g-\bar{g}}{2 i}=\sum_{j=1}^{n-1}\left|f_{j}\right|^{2}+\sum_{j=1}^{N-n}\left|\phi_{j}\right|^{2}, \quad(z, w) \in M_{1} .
$$

Applying $L_{l}, L_{k} L_{l}$ and $\overline{L_{k}} L_{l}$ to $(2.2)$, we have

$$
\frac{L_{l} g}{2 i}=\sum_{j} L_{l} f_{j} \overline{f_{j}}+\sum_{j} L_{l} \phi_{j} \overline{\phi_{j}}
$$




$$
\begin{aligned}
\frac{L_{k} L_{l} g}{2 i}= & \sum_{j} L_{k}\left(L_{l} f_{j}\right) \overline{f_{j}}+\sum_{j} L_{k}\left(L_{l} \phi_{j}\right) \overline{\phi_{j}} \\
\frac{\overline{L_{k}} L_{l g}}{2 i}= & \sum_{j}\left(\overline{L_{k}} L_{l} f_{j} \cdot \overline{f_{j}}+L_{l} f_{j} \cdot \overline{L_{k} f_{j}}\right) \\
& +\sum_{j}\left(\overline{L_{k}} L_{l} \phi_{j} \cdot \phi_{j}+L_{l} \phi_{j} \cdot \overline{L_{k} \phi_{j}}\right) .
\end{aligned}
$$

Letting $(z, w)=(0,0)$ in the above first two equations, we obtain

$$
\left.\frac{\partial g}{\partial z_{l}}\right|_{0}=\left.\frac{\partial^{2} g}{\partial z_{k} \partial z_{l}}\right|_{0}=0
$$

On the other hand, write $\tilde{f}=(f, \phi)$,

$$
E_{l}=\left.\left(\frac{\partial \widetilde{f}}{\partial z_{l}}\right)\right|_{0}=\left.\left(\frac{\partial f_{1}}{\partial z_{l}}, \cdots \frac{\partial f_{n-1}}{\partial z_{l}}, \frac{\partial \phi_{1}}{\partial z_{l}}, \cdots \frac{\partial \phi_{N-n}}{\partial z_{l}}\right)\right|_{0}
$$

and

$$
E_{w}=\left.\left(\frac{\partial \widetilde{f}}{\partial w}\right)\right|_{0}=\left.\left(\frac{\partial f_{1}}{\partial w}, \cdots \frac{\partial f_{n-1}}{\partial w}, \frac{\partial \phi_{1}}{\partial w}, \cdots \frac{\partial \phi_{N-n}}{\partial w}\right)\right|_{0}
$$

Notice that $\left.\frac{\overline{L_{k}} L_{l} g}{2 i}\right|_{0}=\left.\delta_{l}^{k} \frac{\partial g}{\partial w}\right|_{0}=\delta_{l}^{k} \lambda$ with $\delta_{k}^{l}=\left\{\begin{array}{ll}0, & \text { if } k \neq l \\ 1, & \text { if } k=l\end{array}\right.$. Use of $\left(2.2^{*}\right)$ leads to that $E_{l}{\overline{E_{k}}}^{t}=\lambda \delta_{k}^{l}$. Extend $\left\{E_{1} / \sqrt{\lambda}, \cdots, E_{n-1} / \sqrt{\lambda}\right\}$ to a certain orthonormal basis $\left\{E_{1} / \sqrt{\lambda}, \cdots, E_{n-1} / \sqrt{\lambda}, C_{1}, \cdots, C_{N-n}\right\}$ of $\mathbf{C}^{N-1}$. Denote by $A$ the $(N-1) \times(N-1)$ (constant unitary) matrix

$$
A:=\left(\begin{array}{c}
E_{1} / \sqrt{\lambda} \\
\cdot \\
\cdot \\
\cdot \\
E_{n-1} / \sqrt{\lambda} \\
C_{1} \\
\cdot \\
\cdot \\
C_{N-n}
\end{array}\right) .
$$

Let

$$
\begin{aligned}
F^{*}=\left(\widetilde{f^{*}}, g^{*}\right): & =\left(f_{1}{ }^{*}, \cdots, f_{n-1}{ }^{*}, \phi_{1}{ }^{*}, \cdots, \phi_{N-n}{ }^{*}, g^{*}\right) \\
& =\frac{1}{\sqrt{\lambda}} F \cdot\left(\begin{array}{cc}
A^{t} & 0 \\
0 & 1 / \sqrt{\lambda}
\end{array}\right) .
\end{aligned}
$$


Then $F^{*}\left(M_{1}\right) \subset \partial \mathbf{H}_{N}$ and

$$
F^{* \prime}(0)=\left(\begin{array}{ccc}
(\mathrm{id})_{(n-1) \times(n-1)} & (0)_{(n-1) \times(N-n)} & 0 \\
* & * & 1
\end{array}\right) .
$$

Notice that $g=\lambda g^{*}$. One can thus write

$$
\begin{aligned}
f_{j}^{*} & =z_{j}+a_{j} w+\sum_{k, l=1}^{n-1} e_{k l}^{j} z_{k} z_{l}+O\left(|w|^{2}\right)+o\left(|(z, w)|^{2}\right), \\
\phi_{j}^{*} & =b_{j} w+\sum_{k, l=1}^{n-1} q_{k l}^{j} z_{k} z_{l}+O\left(\left|w^{2}\right|+|z w|\right)+o\left(|(z, w)|^{2}\right), \\
g^{*} & =w+d w^{2}+O(|z w|)+o\left(|(z, w)|^{2}\right),
\end{aligned}
$$

where $a_{j}, b_{j}, e_{k l}^{j}, q_{k l}^{j}$ and $d$ are certain constants with $e_{k l}^{j}=e_{l k}^{j}, q_{k l}^{j}=q_{l k}^{j}$. Write

$$
\mathbf{a}=\left(a_{1}, \cdots, a_{n-1}, b_{1}, \cdots, b_{N-n}\right), r=\frac{1}{2} \operatorname{Re}\left\{\left.\frac{\partial^{2} g^{*}}{\partial w^{2}}\right|_{0}\right\},
$$

and define $G \in \operatorname{Aut}_{0}\left(\mathbf{H}_{N}\right)([29,1.2 .1, \mathrm{p} .164])$ by

$$
\left.\begin{array}{rl}
G=( & \frac{z^{*}-\mathbf{a} w^{*}}{1+2 i<z^{*}, \overline{\mathbf{a}}>-(-r+i<\mathbf{a}, \overline{\mathbf{a}}>) w^{*}}, \\
\frac{w^{*}}{1+2 i<z^{*}, \overline{\mathbf{a}}>-(-r+i<\mathbf{a}, \overline{\mathbf{a}}>) w^{*}}
\end{array}\right),
$$

where we have used the standard notation $\langle\alpha, \beta\rangle=\sum_{j=1}^{k} \alpha_{j} \beta_{j}$ for any vectors $\alpha, \beta \in \mathbf{C}^{k}$. Thus by a direct computation, we see that $F^{* *}=\left(\tilde{f}^{* *}, g^{* *}\right):=G \circ F^{*}$ still maps $M_{1}$ into $\partial \mathbf{H}_{N}$. Moreover, $F^{* *}$ satisfies the following normalization condition (compare (2.5) with $[8$, (2.9), p. 231]):

$$
\begin{aligned}
& F^{* *}, \frac{\partial\left(f^{* *}-z\right)}{\partial z_{l}}, \frac{\partial f^{* *}}{\partial w}, \frac{\partial \phi^{* *}}{\partial z_{l}}, \frac{\partial \phi^{* *}}{\partial w} \\
& \frac{\partial g^{* *}}{\partial z_{l}}, \frac{\partial\left(g^{* *}-w\right)}{\partial w}, \frac{\partial^{2} g^{* *}}{\partial z_{l} \partial z_{k}}, \operatorname{Re} \frac{\partial^{2} g^{* *}}{\partial w^{2}}=0, \text { at }(z, w)=0 .
\end{aligned}
$$

Notice

$$
\begin{aligned}
& a_{l}=\frac{1}{\lambda} E_{w} \cdot{\overline{E_{l}}}^{t}, b_{l}=\frac{1}{\sqrt{\lambda}} E_{w} \cdot \bar{C}_{l}^{t} \\
& e_{k l}^{j}=\frac{1}{2 \lambda} \widetilde{f}_{z_{k} z_{l}}^{\prime \prime}(0) \cdot{\overline{E_{j}}}^{t}, q_{k l}^{j}=\frac{1}{2 \sqrt{\lambda}} \widetilde{f}_{z_{k} z_{l}}^{\prime \prime}(0) \cdot \bar{C}_{j}^{t} .
\end{aligned}
$$


We have the following, which will be used for our proof of Theorem A.

Lemma 2.1. Assume the notation which we have set up so far. Suppose that

$$
f^{* *}=z+O\left(|w|^{2}+|z w|\right)+o\left(|(z, w)|^{2}\right)
$$

and

$$
\phi^{* *}=O\left(|w|^{2}+|z w|\right)+o\left(|(z, w)|^{2}\right) .
$$

Namely, suppose that $\left.\frac{\partial^{2} f^{* *}}{\partial z_{k} z_{j}}\right|_{0}=\left.\frac{\partial^{2} \phi^{* *}}{\partial z_{k} z_{j}}\right|_{0}=0$ for any $k, j$. Then one has the following feedback for $\widetilde{f}$ :

$$
\widetilde{f}_{z_{k} z_{l}}^{\prime \prime}(0)=\frac{2 \sqrt{-1}}{\lambda}\left(\overline{E_{w}} \cdot E_{l}^{t}\right) E_{k}+\frac{2 \sqrt{-1}}{\lambda}\left(\overline{E_{w}} \cdot E_{k}^{t}\right) E_{l}
$$

Proof of Lemma 2.1. Notice that

$$
\phi_{j}^{* *}=\frac{\phi_{j}^{*}-b_{j} g^{*}}{1+2 i<\widetilde{f^{*}}, \overline{\mathbf{a}}>-(-r+i<\mathbf{a}, \overline{\mathbf{a}}>) g^{*}} .
$$

Comparing the coefficients of terms of the form $z_{l} z_{k}$ in the Taylor expansion of the above expression and using the given hypothesis, we get easily that $q_{k l}^{j}=0$, for any $j, k, l$. Since $q_{k l}^{j}=\frac{1}{2 \sqrt{\lambda}} \widetilde{f}_{z_{l} z_{k}}^{\prime \prime}(0) \cdot \bar{C}_{j}^{t}$, it follows that the $(N-1)$-tuple $\widetilde{f}_{z_{l} z_{k}}^{\prime \prime}(0)$ stays in the space spanned by $\left\{E_{1}, \cdots, E_{n-1}\right\}$, so that there exist constants $\left\{\lambda_{k l}^{j}\right\}$ such that

$$
\widetilde{f}_{z_{k} z_{l}}^{\prime \prime}(0)=\sum_{j=1}^{n-1} \lambda_{k l}^{j} E_{j}
$$

Next, considering the Taylor expansion of $f_{j}^{* *}$ in the following

$$
f_{j}^{* *}=\frac{f_{j}^{*}-a_{j} g^{*}}{1+2 i<\widetilde{f^{*}}, \overline{\mathbf{a}}>-(-r+i<\mathbf{a}, \overline{\mathbf{a}}>) g^{*}}
$$

and using the hypothesis, we obtain $\sum e_{k l}^{j} z_{k} z_{l}-2 i \sum_{l} \overline{a_{l}} z_{j} z_{l} \equiv 0$. Hence, it follows that $e_{k l}^{j}=\sqrt{-1}\left(\delta_{k}^{j} \overline{a_{l}}+\delta_{l}^{j} \overline{a_{k}}\right)$, so that, by (2.6),

$$
\frac{1}{2 \lambda} \tilde{f}_{z_{k} z_{l}}^{\prime \prime}(0) \overline{E_{j}}{ }^{t}=\frac{\sqrt{-1} \delta_{k}^{j}}{\lambda} \overline{E_{w}} \cdot E_{l}^{t}+\frac{\sqrt{-1} \delta_{l}^{j}}{\lambda} \overline{E_{w}} \cdot E_{k}^{t} .
$$


Combing this with (2.7) and making use of the orthogonality: $E_{l} \cdot{\overline{E_{j}}}^{t}=$ $\lambda \delta_{l}^{j}$ gives

$$
\lambda_{k l}^{j}=\frac{2 \sqrt{-1}}{\lambda}\left(\delta_{k}^{j} \overline{E_{w}} \cdot E_{l}^{t}+\delta_{l}^{j} \overline{E_{w}} \cdot E_{k}{ }^{t}\right) .
$$

Returning to $(2,7)$ completes the proof of Lemma 2.1. q.e.d.

\section{Chern-Moser Lie-derivate and a semi-linear equation}

In this and the following sections, we will present the proof of Theorem A. After the discussion in $\S 2$, the $C^{2}$-smooth CR map $F=(f, \phi, g)$ sending $M_{1}$ into $M_{2}$ can, now and in what follows, be assumed to take the following form:

$$
\begin{aligned}
& f=z+\hat{f}, g=w+\hat{g} \text { with } \hat{f}, \hat{g}, \phi=O\left(|(z, w)|^{2}\right), \\
& \left.\frac{\partial^{2} \hat{g}}{\partial z_{l} \partial z_{k}}\right|_{0},\left.\operatorname{Re} \frac{\partial^{2} \hat{g}}{\partial w^{2}}\right|_{0}=0 .
\end{aligned}
$$

Our idea for the proof of Theorem A can be briefly described as follows: We first use the Moser formal theory to verify the hypothesis in Lemma 2.1. Then, we compose $f$ with the automorphisms of the Heisenberg hypersurfaces to produce a continuous family of mappings, from which we will derive a differential equation (See $\left(4.1^{\prime}\right)$ in $\S 4$ ). This equation will be good enough to tell that $F=(z, 0, w)$. In this section, we will extend the lower order case of a fundamental lemma of ChernMoser to the case applicable in our setting.

To proceed, we first recall some notation and definitions used in [8] and [25], and assign the weights 1 and 2 to $z$ and $w$, respectively. Then a (possibly real) polynomial $h(z, w, \bar{z}, \bar{w})$ is a weighted homogeneous polynomial of degree $s$ if for each $t>0, h\left(t z, t^{2} w, t \bar{z}, t^{2} \bar{w}\right)=t^{s} h(z, w, \bar{z}, \bar{w})$. We say that a function $h$ defined over $M_{1}$ is in the class $\mathcal{P}+o_{w t}(s)$ if there is a polynomial $h_{1}$ and a function $h_{2}$ such that $h=h_{1}+h_{2}$ with $\lim _{t \rightarrow 0^{+}} \frac{h_{2}\left(t z, t^{2} w, t \bar{z}, t^{2} \bar{w}\right)}{t^{s}} \rightarrow 0$ uniformly with respect to $(z, w) \approx\left(0^{\prime}, 0\right)$. When the above $h_{1} \equiv 0$, we say $h \in o_{w t}(s)$.

Now, substituting (3.1) into (2.2), we obtain

$$
\operatorname{Im}(w+\hat{g})=\sum_{j=1}^{n-1}\left|z_{j}+\hat{f}_{j}\right|^{2}+\sum_{j=1}^{N-n}\left|\phi_{j}\right|^{2}, \quad(z, w) \in M_{1} .
$$

Suppose that we have the decompositions: $\hat{f}_{j}=\sum_{s=2}^{m-1} \hat{f}_{j}^{(s)}+o_{w t}(m-1)$, $\hat{g}=\sum_{s=2}^{m} \hat{g}^{(s)}+o_{w t}(m)$, and $\phi_{j}=\sum_{s=l}^{m-l} \phi_{j}^{(s)}+o_{w t}(m-l)($ with $l \geq 2)$. 
Here, as in [25], we use the notation $(\cdot)^{(\sigma)}$ to denote a certain weighted holomorphic homogeneous polynomial of weighted degree $\sigma$. Then, after collecting terms in (3.2) of weighted degree $k$, we have the following $k$-th semi-linearization of $(3.2)$ :

$$
\begin{aligned}
\operatorname{Im}\left(\hat{g}^{(k)}(z, w)-2 i<\bar{z}, \hat{f}^{(k-1)}(z, w)>\right) \\
\quad=\sum_{j=1}^{N-n} \sum_{p=l}^{k-l} \phi_{j}^{(k-p)} \bar{\phi}_{j}^{(p)}+N^{(k)}, \quad(z, w) \in M_{1},
\end{aligned}
$$

where $m \geq k \geq l$, and $N^{(k)}$ is a weighted homogeneous polynomial of weighted degree $k$, contributed by $\hat{f}^{(\sigma-1)}$ with $\sigma \leq k-1$. Moreover, $N^{(k)} \equiv 0$ if $\hat{f}^{(\sigma-1)} \equiv 0$ for $\sigma \leq k-1$. Therefore, when $\hat{g}^{\left(\sigma_{1}\right)}, \hat{f}^{\left(\sigma_{1}-1\right)}$, $\phi^{\left(\sigma_{2}\right)} \equiv 0$ for $\sigma_{1}<2 k$ and $\sigma_{2}<k(2 k \leq m)$, the $2 k$-th linearization of (3.2) can be simplified as

$$
\begin{aligned}
\operatorname{Im}\left(\hat{g}^{(2 k)}(z, w)\right. & \left.-2 i<\bar{z}, \hat{f}^{(2 k-1)}(z, w)>\right) \\
& =\sum_{j=1}^{N-n} \phi_{j}^{(k)} \bar{\phi}_{j}^{(k)}, \quad(z, w) \in M_{1} .
\end{aligned}
$$

Similarly, when $\hat{g}^{\left(\sigma_{1}\right)}, \hat{f}^{\left(\sigma_{1}-1\right)}, \phi^{\left(\sigma_{2}\right)} \equiv 0$ for $\sigma_{1} \leq 2 k$ and $\sigma_{2} \leq k(2 k+1 \leq$ $m)$, the $(2 k+1)$-th linearization of $(3.2)$ reduces to

$$
\begin{array}{r}
\operatorname{Im}\left(\hat{g}^{(2 k+1)}(z, w)-2 i<\bar{z}, \hat{f}^{(2 k)}(z, w)>\right)=0, \\
(z, w) \in M_{1} .
\end{array}
$$

Write $\mathcal{L}\left(\hat{f}^{(\sigma-1)}, \hat{g}^{(\sigma)}\right)=\left.\operatorname{Im}\left(\hat{g}^{(\sigma)}-2 i<\bar{z}, \hat{f}^{(\sigma-1)}>\right)\right|_{M_{1}}$, which is the Lie derivative of $\rho_{1}=\operatorname{Im} w-\sum_{j=1}^{n-1}\left|z_{j}\right|^{2}$ along the vector field $X=2 \operatorname{Re}\left(\hat{g}^{(\sigma)} \frac{\partial}{\partial w}+\sum \hat{f}_{j}{ }^{(\sigma-1)} \frac{\partial}{\partial z_{j}}\right)$ restricted to $M_{1}$. The operator $\mathcal{L}$ is the basic tool for the construction of the local normal form of strongly pseudoconvex hypersurfaces, and for many other studies (see, for instance, the work [8], [29], etc). In particular, we mention the following lemma of Chern-Moser, which partially motivated our present study:

Lemma 3.0 ([8, Lemma 2.1, p.233]). Consider the linear equation $\mathcal{L}(f, g)=\operatorname{Im}(g-2 i<\bar{z}, f>)=0$ with $f, g$ holomorphic near the origin. Then it has a unique solution $(f, g) \equiv(0,0)$ if the following normalization condition holds:

$$
\left.f\right|_{0},\left.g\right|_{0},\left.\frac{\partial f}{\partial w}\right|_{0},\left.\frac{\partial f}{\partial z_{l}}\right|_{0},\left.\frac{\partial g}{\partial z_{l}}\right|_{0},\left.\frac{\partial g}{\partial w}\right|_{0}, \frac{\partial^{2} g}{\left.\partial z_{l} z_{k}\right|_{0}},\left.\operatorname{Re}\left(\frac{\partial^{2} g}{\partial w^{2}}\right)\right|_{0}=0
$$


For the proof of Theorem A, the following lemma involving $\mathcal{L}$ is of fundamental importance:

Proposition 3.1. Consider the following semi-linear equation of weighted degree 4 with respect to the holomorphic polynomials $P^{(4)}$, $Q^{(3)}=\left(Q_{1}^{(3)}, \cdots, Q_{n-1}^{(3)}\right)$, and $\Phi_{j}^{(2)}(j=1, \cdots, k)$ of weighted degree 4,3 , and 2 , respectively:

$$
\mathcal{L}\left(Q^{(3)}, P^{(4)}\right)=\sum_{j=1}^{k}\left|\Phi_{j}^{(2)}(z, w)\right|^{2}, \quad(z, w) \in M_{1} .
$$

Assume the following normalization condition holds:

$$
\frac{\partial \Phi_{j}^{(2)}}{\partial w}=\operatorname{Re} \frac{\partial^{2} P^{(4)}}{\partial w^{2}}=0 .
$$

Then $P^{(4)} \equiv 0, Q^{(3)}=a^{(1)}(z)$ w with

$$
-2 i<a^{(1)}(z), \bar{z}>|z|^{2}=\sum_{j=1}^{k}\left|\Phi_{j}^{(2)}(z)\right|^{2},
$$

where $a^{(1)}(z)$ is a vector-valued holomorphic polynomial of degree one. Moreover, if $k \leq n-2$, then (3.4) has only trivial solution $Q_{j}^{(3)}=P^{(4)}=$ $\Phi_{j}^{(2)} \equiv 0$.

We should mention that when all $\Phi_{j}^{(2)}$ are assumed to be 0 , then Proposition 3.1 reduces to $4^{\text {th }}$-order case of Lemma 3.0. Also, we point out that when $k \geq n-1$, The last statement in Proposition 3.1 fails. Indeed, this is the only place in the course of the proof of Theorem A, where the codimension restriction is used. The geometric interpretation of Proposition 3.1 is that the expression $\sum_{j=1}^{k}\left|\Phi_{j}^{(2)}\right|^{2}$ stays in the Moser normal space $\mathcal{N}^{4}$ ([8]) when $k \leq n-2$. We remark that the last statement in Proposition 3.1 may hold also in any higher degree case. However, we will not pursue this because the present version of Proposition 3.1 is good enough for our purpose later.

Proof of Proposition 3.1. By $\left(3.4^{\prime}\right)$, we can write

$$
Q^{(3)}(z, w)=a^{(1)}(z) w+b^{(3)}(z)
$$




$$
P^{(4)}(z, w)=A^{(4)}(z)+B^{(2)}(z) w+D_{0} w^{2}
$$

with $\operatorname{Re}\left(D_{0}\right)=0$, and $\Phi_{j}^{(2)}(z, w)=h_{j}^{(2)}(z)$. Here $a^{(1)}$ and $b^{(3)}$ are vectorvalued holomorphic polynomials of degrees 1 and 3 , respectively. Then (3.4) reads as

$$
\begin{gathered}
\operatorname{Im}\left(A^{(4)}+B^{(2)} w+D_{0} w^{2}-2 i<\bar{z}, a^{(1)}>w-2 i<\bar{z}, b^{(3)}>\right) \\
=\sum_{j=1}^{k}\left|h_{j}^{(2)}(z)\right|^{2}, \quad(z, w) \in M_{1},
\end{gathered}
$$

where $w=u+i|z|^{2}$ with $u=\operatorname{Re}(w)$. Noticing that $\{z, u\}$ are independent variables, $\left(3.5^{\prime}\right)$ can be split into the following equations:

$$
\operatorname{Im}\left(A^{(4)}(z)+i B^{(2)}(z)|z|^{2}-D_{0}|z|^{4}+2<\bar{z}, a^{(1)}(z)|z|^{2}-i b^{(3)}(z)>\right)
$$

$$
=\sum_{j=1}^{k}\left|h_{j}^{(2)}(z)\right|^{2}
$$

$$
\begin{aligned}
\operatorname{Im}\left(B^{(2)}(z)+2 i D_{0}|z|^{2}-2 i<\bar{z}, a^{(1)}(z)>\right) u & =0 \\
\operatorname{Im}\left(D_{0} u^{2}\right) & =0 .
\end{aligned}
$$

Now, $\left(3.5^{\prime \prime \prime}\right)$ together with the normalization condition gives that $D_{0}=$ 0 . Moreover, noting that $u$ is real and collecting terms of the form $z_{l} z_{k}$, we can also conclude from $\left(3.5^{\prime \prime \prime}\right)$ that $B^{(2)} \equiv 0$. Hence

$$
\operatorname{Im}\left(i<\bar{z}, a^{(1)}(z)>\right) \equiv 0 .
$$

Returning to $\left(3.5^{\prime \prime}\right)$, we similarly obtain that $A^{(4)} \equiv 0$ and $b^{(3)} \equiv 0$. Therefore, $P^{(4)} \equiv 0$ and $Q^{(3)}=a^{(1)}(z) w$. Furthermore, combining these with $\left(3.5^{\prime \prime}\right)$, we get

$$
-2 i<\bar{z}, a^{(1)}(z)>|z|^{2}=\sum_{j=1}^{k}\left|h_{j}^{(2)}(z)\right|^{2} .
$$

To complete the proof of the second part of Proposition 3.1, it now suffices for us to apply the following Lemma 3.2 to the equation $-2 i<$ $\bar{z}, a^{(1)}(z)>|z|^{2}=\sum_{j=1}^{k}\left|h_{j}^{(2)}(z)\right|^{2}$ : 
Lemma 3.2. Let $\left\{\psi_{j}\right\}_{j=1}^{k}$ and $\left\{\chi_{j}\right\}_{j=1}^{k}$ be holomorphic functions in $z \in \mathbf{C}^{n}$ near the origin. Assume that $\psi_{j}(0)=\chi_{j}(0)=0$. Let $H(z, \bar{z})$ be a real analytic function for $z \approx 0$ such that

$$
\sum_{j=1}^{k} \psi_{j}(z) \overline{\chi_{j}(z)}=|z|^{2} H(z, \bar{z}) .
$$

Then when $k \leq n-1, H(z, \bar{z}) \equiv 0$.

Proof of Lemma 3.2. Complexifying (3.6), we have

$$
\sum_{j=1}^{k} \psi_{j}(z) \overline{\chi_{j}(\xi)}=<z, \bar{\xi}>H(z, \bar{\xi})
$$

with $z, \xi$ independent variables. Without loss of generality, we can further assume that $\psi_{j} \not \equiv 0$ for each $j$. Therefore, we can find a point $z_{0}$ sufficiently close to the origin such that $\psi_{j}\left(z_{0}\right)=\epsilon_{j} \neq 0$ for each $j$.

By the assumption: $k \leq n-1$, we can easily see that

$$
V_{z_{0}}=\left\{z: \psi_{j}(z)=\psi_{j}\left(z_{0}\right), j=1, \cdots, k\right\}
$$

defines a complex analytic variety of dimension at least 1 near $z_{0}$. By our choice of $z_{0}$ and by $\psi_{j}(0)=0$, it is clear that $V_{z_{0}}$ can not contain a complex line passing through the origin. Hence, there exists a point $z^{*} \in V_{z_{0}}$ such that $V_{z_{0}}$ contains a complex curve $C^{*}$ near $z^{*}$ parametrized by an equation of the form

$$
z(t)=z^{*}+v t+o(t)
$$

where $\left\{z^{*}, v\right\}$ are independent vectors and $|t|<1$. Notice that for each $z \in C^{*}$ and $\xi$ with $\langle z, \bar{\xi}\rangle=0$ and $\xi$ close to 0 , we have $\sum \overline{\epsilon_{j}} \chi_{j}(\xi)=0$. Also notice that a direction computation based on (3.7) shows that all such $\xi$ fill in an open subset of $\mathbf{C}^{n}$. We conclude that $\sum_{j} \bar{\epsilon}_{j} \chi_{j}(z) \equiv 0$. Hence, (3.6) can be reduced to

$$
\sum_{j=1}^{k-1}\left(\psi_{j}(z)-\frac{\epsilon_{j}}{\epsilon_{k}} \psi_{k}(z) \overline{\left(\chi_{j}(z)\right)}=<z, \bar{z}>H(z, \bar{z}) .\right.
$$

Now, applying an induction argument, it follows easily that $\sum \psi_{j} \chi_{j} \equiv 0$ and $H \equiv 0$. q.e.d. 


\section{A criterion for linearity}

We now give a criterion on linearity for $C^{2}$-smooth CR mappings between spheres. Notice that the results of the present section applies to any codimensional case. Namely, $N$ can be any positive integer with $N \geq n$.

To start, we let $T=\frac{\partial}{\partial u}$ be the real tangent vector field along $\partial \mathbf{H}_{n}$ transversal to $\mathrm{T}^{(1,0)} \partial \mathbf{H}_{n}+\mathrm{T}^{(0,1)} \partial \mathbf{H}_{n}$. We notice that

$$
\left\{L_{1}, \cdots, L_{n-1}, \overline{L_{1}}, \cdots, \overline{L_{n-1}}, T\right\} \text { forms a basis of } C \mathrm{~T} \partial \mathbf{H}_{n} \text {. }
$$

Parametrize $M_{1}$ by $\mathbf{R}^{2 n-1}$ through $(z, u) \rightarrow(z, w)=\left(z, u+i|z|^{2}\right)$, and introduce the standard pairing $(\cdot, \cdot)$ over $M_{1}$. For any differential operator $Y$ with smooth coefficients along $M_{1}$, we can find its formal adjoint differential operator $Y^{*}$ with smooth coefficients such that $(Y(\chi), \rho)=$ $\left(\chi, Y^{*}(\rho)\right)$. Here $\chi \in C^{\infty}\left(M_{1}\right), \rho \in C_{0}^{\infty}\left(M_{1}\right)$. Then for each continuous function $h$ and a differential operator with smooth coefficients $Y$ along $M_{1}, Y(h)$ can be identified as a distribution acting on the testing function space $C_{0}^{\infty}\left(M_{1}\right)$ in the canonical way: $(Y(h), \rho)=\left(h, Y^{*}(\rho)\right)$. A continuous function $h$ is called a CR function if $\bar{L}_{j}(h) \equiv 0$ in the sense of distribution for each $j$.

With the above notion, we can state the following useful fact, whose proof is quite straightforward:

Lemma 4.1. (a) $T$ commutes with $L_{j}$ for each $j$. Hence, for any continuous $C R$ function $h$ over $M_{1}$, Th is a CR distribution over $M_{1}$, too. Moreover, $T h=-\frac{1}{2 i}\left[L_{j}, \overline{L_{j}}\right] h=\frac{1}{2 i} \overline{L_{j}}\left(L_{j} h\right)$.

(b) Let $h$ be a $C^{2} C R$ function over $M_{1}$ and let $\chi$ be a $C^{1}$ function over $M_{1}$. Then for any $k$, it holds, in the sense of distribution that

$$
\begin{aligned}
& \overline{L_{k}}\left(L_{k}^{2}(h) \chi\right)=4 i L_{k}(T(h)) \chi+L_{k}^{2}(h) \overline{L_{k}}(\chi) \\
& \overline{L_{k}}\left(L_{k}(T(h)) \chi\right)=2 i T^{2}(h) \chi+L_{k}(T(h)) \overline{L_{k}}(\chi) .
\end{aligned}
$$

Next, let $F$ be a twice continuously differentiable CR mapping from $M_{1}$ into $M_{2}$ such that $F(0)=0$, which satisfies the normalization in (3.1). For each $\left(z_{0}, w_{0}\right) \in M_{1}$, write $\sigma_{\left(z_{0}, w_{0}\right)} \in \operatorname{Aut}\left(\mathbf{H}_{n}\right)$ for the map sending $(z, w)$ to $\left(z+z_{0}, w+w_{0}+2 i<z, \overline{z_{0}}>\right)$. Define $\tau_{\left(z_{0}, w_{0}\right)} \in$ $\operatorname{Aut}\left(\mathbf{H}_{N}\right)$ by

$$
\tau\left(z^{*}, w^{*}\right)=\left(z^{*}-\tilde{f}\left(z_{0}, w_{0}\right), w^{*}-\overline{g\left(z_{0}, w_{0}\right)}-2 i<z^{*}, \overline{\widetilde{f}\left(z_{0}, w_{0}\right)}>\right),
$$


where $\tilde{f}=(f, \phi)$ is as before. Then

$$
F_{\left(z_{0}, w_{0}\right)}(z, w)=\left(f_{\left(z_{0}, w_{0}\right)}, \phi_{\left(z_{0}, w_{0}\right)}, g_{\left(z_{0}, w_{0}\right)}\right)=\tau_{\left(z_{0}, w_{0}\right)} \circ F \circ \sigma_{\left(z_{0}, w_{0}\right)}
$$

is still a twice continuously differentiable CR map from a small open piece of $\partial \mathbf{H}_{n}$ containing 0 into $\partial \mathbf{H}_{N}$ with $F_{\left(z_{0}, w_{0}\right)}(0)=0$. As in $\S 2$, we can similarly define the data $\lambda_{\left(z_{0}, w_{0}\right)},\left(E_{l}\right)_{\left(z_{0}, w_{0}\right)},\left(E_{w}\right)_{\left(z_{0}, w_{0}\right)},\left(C_{l}\right)_{\left(z_{0}, w_{0}\right)}$, $A_{\left(z_{0}, w_{0}\right)}, F_{\left(z_{0}, w_{0}\right)}^{*}, a_{\left(z_{0}, w_{0}\right)}, r_{\left(z_{0}, w_{0}\right)},\left(e_{k l}^{j}\right)_{\left(z_{0}, w_{0}\right)},\left(q_{k l}^{j}\right)_{\left(z_{0}, w_{0}\right)}$, and $G_{\left(z_{0}, w_{0}\right)}$, etc, which are now subscripted by $\left(z_{0}, w_{0}\right)$ for they depend on the choice of $\left(z_{0}, w_{0}\right)$. As in $\S 2$, we can normalize $F_{\left(z_{0}, w_{0}\right)}^{*}$ by composing it with $G_{\left(z_{0}, w_{0}\right)}$ to get the map $F_{\left(z_{0}, w_{0}\right)}^{* *}=\left(f_{\left(z_{0}, w_{0}\right)}^{* *}, \phi_{\left(z_{0}, w_{0}\right)}^{* *}, g_{\left(z_{0}, w_{0}\right)}^{* *}\right)$ so that the normalization condition as in $(2.5)$ holds for $F_{\left(z_{0}, w_{0}\right)}^{* *}$. Notice that $F_{\left(z_{0}, w_{0}\right)}^{* *}$ also depends on the choice of $\left(C_{l}\right)_{\left(z_{0}, w_{0}\right)}$. The purpose of this section is to prove the following linearity criterion, which should be also useful for other related investigations.

Theorem 4.2. Let $F$ be a twice continuously differentiable $C R$ mapping from $M_{1}$ into $M_{2}$ such that $F(0)=0$, which satisfies the normalization in (3.1). Assume the new map $F_{\left(z_{0}, w_{0}\right)}^{* *}$ is as defined in the above paragraph. Suppose that for each $\left(z_{0}, w_{0}\right)(\approx 0) \in M_{1}$, it always holds that

$$
f_{\left(z_{0}, w_{0}\right)}^{* *}=z+O\left(|w|^{2}+|z||w|\right)+o\left(|(z, w)|^{2}\right)
$$

and

$$
\phi_{\left(z_{0}, w_{0}\right)}^{* *}=O\left(|w|^{2}+|z||w|\right)+o\left(|(z, w)|^{2}\right) .
$$

Then $F(z, w) \equiv(z, 0, w)$.

We remark that in terms of Lemma 5.3 to be presented in the next section, the assumption in Theorem 4.2 is equivalent to that $\left.\frac{\partial^{2}\left(\phi_{\left(z_{0}, w_{0}\right)}^{* *}\right)}{\partial z_{k} \partial z_{l}}\right|_{0}$ $=0$, or that $\left.\frac{\partial^{2}\left(f_{\left(z_{0}, w_{0}\right)}^{* *}\right)}{\partial z_{k} \partial w}\right|_{0}=0$ for each $k, l$ and $\left(z_{0}, w_{0}\right) \approx 0$.

Before proceeding to the proof of Theorem 4.2, we need to put Lemma 2.1 in a more applicable form. To this aim, we first write $z_{0}=\left(z_{1,0}, \cdots, z_{n-1,0}\right)$, and

$$
\tilde{f}_{\left(z_{0}, w_{0}\right)}=\left(\left(f_{1}\right)_{\left(z_{0}, w_{0}\right)}, \cdots,\left(f_{n-1}\right)_{\left(z_{0}, w_{0}\right)},\left(\phi_{1}\right)_{\left(z_{0}, w_{0}\right)}, \cdots,\left(\phi_{N-n}\right)_{\left(z_{0}, w_{0}\right)}\right)
$$

In the following, we first collect certain expressions whose proofs follow 
from a direct computation:

$$
\begin{aligned}
\left(E_{w}\right)_{\left(z_{0}, w_{0}\right)}= & \left(\widetilde{f}_{\left(z_{0}, w_{0}\right)}\right)_{w}^{\prime}(0)=\widetilde{f}_{w}^{\prime}\left(z_{0}, w_{0}\right) \\
\left(E_{l}\right)_{\left(z_{0}, w_{0}\right)}= & \left(\widetilde{f}_{\left(z_{0}, w_{0}\right)}\right)_{z_{l}}^{\prime}(0)=\widetilde{f}_{z_{l}}^{\prime}\left(z_{0}, w_{0}\right)+2 i \overline{z_{l, 0}} \widetilde{f}_{w}^{\prime}\left(z_{0}, w_{0}\right) \\
= & L_{l}(\widetilde{f})\left(z_{0}, w_{0}\right) \\
\left(g_{\left(z_{0}, w_{0}\right)}\right)_{z_{l}}^{\prime}(0)= & L_{l}(g-2 i<\widetilde{f}, \overline{\widetilde{f}}>)\left(z_{0}, w_{0}\right) \\
\left(g_{\left(z_{0}, w_{0}\right)}\right)_{w}^{\prime}(0)= & g_{w}^{\prime}\left(z_{0}, w_{0}\right)-2 i<\widetilde{f}_{w}^{\prime}\left(z_{0}, w_{0}\right), \overline{\widetilde{f}\left(z_{0}, w_{0}\right)}> \\
\lambda\left(z_{0}, w_{0}\right):= & \lambda_{\left(z_{0}, w_{0}\right)}=g_{w}^{\prime}\left(z_{0}, w_{0}\right)-2 i<\widetilde{f}_{w}^{\prime}\left(z_{0}, w_{0}\right), \overline{\widetilde{f}\left(z_{0}, w_{0}\right)}>; \\
\left(\widetilde{f}_{\left(z_{0}, w_{0}\right)}\right)_{z_{l} z_{k}}^{\prime \prime}(0)= & (\widetilde{f})_{z_{k} z_{l}}^{\prime \prime}\left(z_{0}, w_{0}\right)+2 i \overline{z_{0, l}}(\widetilde{f})_{z_{k} w}^{\prime \prime}\left(z_{0}, w_{0}\right) \\
& +2 i \overline{z_{0, k}}(\widetilde{f})_{z_{l} w}^{\prime \prime}\left(z_{0}, w_{0}\right)-4 \overline{z_{0, l} z_{0, k}}(\widetilde{f})_{w w}^{\prime \prime}\left(z_{0}, w_{0}\right) \\
= & L_{l}\left(L_{k}(\widetilde{f})\right)\left(z_{0}, w_{0}\right) .
\end{aligned}
$$

Noticing the arbitrariness of $\left(z_{0}, w_{0}\right)$ and applying Lemma 2.1 to $F_{\left(z_{0}, w_{0}\right)}^{* *}$, the above formulas then allow Lemma 2.1 to be restated as follows:

Lemma 4.3. Assume the above notation and assume the hypothesis in Theorem 4.2. For each $j, k$ and at each point $(z, w)\left(\in M_{1}\right) \approx 0$, we then have the following:

$$
\begin{aligned}
L_{j}\left(L_{k}(\widetilde{f})\right)= & \frac{2 i}{\lambda(z, w)}\left(\overline{\widetilde{f}}_{w}^{\prime} \cdot\left(L_{j} \widetilde{f}\right)^{t}\right) L_{k}(\widetilde{f}) \\
& +\frac{2 i}{\lambda(z, w)}\left(\overline{\widetilde{f}}_{w}^{\prime} \cdot\left(L_{k} \widetilde{f}\right)^{t}\right) L_{j}(\widetilde{f}) .
\end{aligned}
$$

Proof of Theorem 4.2. Write $A_{k}(z, w)=\frac{2 i \overline{\widetilde{f}_{w}^{\prime}} \cdot L_{k}(\tilde{f})^{t}}{\lambda(z, w)}$, which is clearly $C^{1}$ over $M_{1}$. Assume, without loss of generality that $L_{k}\left(f_{k}\right) \neq 0$ over $M_{1}$. Then we first notice that Lemma 4.3 gives immediately that

$$
\begin{aligned}
A_{k}(z, w) & =\frac{L_{k}^{2}\left(f_{k}\right)}{2 L_{k}\left(f_{k}\right)}, \\
L_{j}\left(L_{k}(\phi)\right) & =A_{j}(z, w) L_{k}(\phi)+A_{k}(z, w) L_{j}(\phi) .
\end{aligned}
$$

In particular, we obtain

$$
L_{k}^{2}(\phi)=2 A_{k}(z, w) L_{k}(\phi) .
$$

In the following, we will often use the simple fact that two continuous functions are the same in the usual sense if and only if they are the same 
in the distribution sense. Now, Applying $\overline{L_{k}}$ to (4.3) and using Lemma 4.1(b), we can easily get

$$
4 i L_{k}(T(\phi))=B_{k, 1}(z, w) L_{k}(\phi)+B_{k, 2} T(\phi),
$$

where $B_{k, 2}=4 i A_{k}(z, w) \in C^{1}\left(M_{1}\right)$ and $B_{k, 1}=\overline{L_{k}}\left(2 A_{k}(z, w)\right) \in C^{0}\left(M_{1}\right)$. Notice that there are certain functions $b_{k, j}$ such that

$$
B_{k, 1}=b_{k, 1}(z, w) L_{k}\left(T\left(f_{k}\right)\right)+b_{k, 2}(z, w) L_{k}^{2}\left(f_{k}\right),
$$

where $b_{k, j}(z, w) \in C^{1}\left(M_{1}\right)$ for $j=1,2$. Indeed, by Lemma (4.2)(b) and (4.2), we have

$$
\overline{L_{k}}\left(A_{k}\right)=\overline{L_{k}}\left(\frac{1}{2 L_{k}\left(f_{k}\right)}\right) L_{k}^{2}\left(f_{k}\right)+\frac{2 i}{L_{k}\left(f_{k}\right)} L_{k}\left(T\left(f_{k}\right)\right)
$$

Hence, to see $\left(4.4^{\prime}\right)$, it suffices to take $b_{k, 2}(z, w)=\overline{L_{k}}\left(\frac{1}{L_{k}\left(f_{k}\right)}\right)$ and $b_{k, 1}=$ $\frac{2 i}{L_{k}\left(f_{k}\right)}$, which are apparently $C^{1}$ over $M_{1}$.

Applying $\overline{L_{k}}$ to (4.4), we obtain in the sense of distribution

$$
-8 T^{2}(\phi)=h+\overline{L_{k}}\left(B_{k, 2}\right)(T(\phi))
$$

where $h=\overline{L_{k}}\left(\left(b_{k, 1} L_{k}\left(T\left(f_{k}\right)\right)+b_{k, 2} L_{k}^{2}\left(f_{k}\right)\right) L_{k}(\phi)\right)$. By Lemma 4.1 (b), $h$ can be identified with

$$
\begin{gathered}
\left.\left(c_{k, 1} L_{k}\left(T\left(f_{k}\right)\right)+c_{k, 2} T^{2}\left(f_{k}\right)+c_{k, 3} L_{k}^{2}\left(f_{k}\right)\right)\right) L_{k}(\phi) \\
+\left(c_{k, 4} L_{k}\left(T\left(f_{k}\right)\right)+c_{k, 5} L_{k}^{2}\left(f_{k}\right)\right) T(\phi)
\end{gathered}
$$

for certain $c_{k, j}^{\prime} \mathrm{s}$, which are continuous over $M_{1}$. Hence, $h$ is equivalent to a continuous function and we thus obtain point-wisely that

$$
T^{2}(\phi)=C_{k, 1}(z, w) L_{k}(\phi)+C_{k, 2}(z, w) T(\phi),
$$

where $C_{k, j}$ are certain continuous functions over $M_{1}$.

Now, since $\phi$ are CR over $M_{1}, \overline{L_{k}} T(\phi)$ and $\overline{L_{j}}\left(\overline{L_{k}}(\phi)\right) \equiv 0$. Identify $M_{1}$ with a neighborhood of 0 in $\mathbf{R}^{2 n-1}$ through the map $(x, y, u) \rightarrow$ $\left(z=(x+i y), w=u+i|z|^{2}\right)$ and write $X$ for the vector $\left(\frac{\partial \phi}{\partial x}, \frac{\partial \phi}{\partial y}, \frac{\partial \phi}{\partial u}\right)$. We then see from (4.0), (4.2), (4.4) and (4.6) that there is a certain matrix $\Lambda(x, y, u)$ whose entries are continuous functions over $M_{1}$ such that $X$ satisfies the following

$$
D X=\Lambda(x, y, u) X^{t}, \quad X(0)=0 .
$$


Regarding (4.7) as a differential equation in $X$ and applying the uniqueness theory, it follows that $X \equiv 0$. Indeed, for any $p=\left(x_{0}, y_{0}, u_{0}\right) \approx$ 0 , let $X_{p}(t)=X(t p)$ for $0 \leq t \leq 1$. Then $\frac{d X_{p}}{d t}=\Lambda_{p}(t) X_{p}(t)^{t}$ by (4.7) for a certain continuous matrix function (in $t \in[0,1]) \Lambda_{p}(t)$ with $\left\|\Lambda_{p}(t)\right\|<\|p\|$, where $\|\cdot\|$ stands for the super-norm. Since $X_{p}(0)=0$,

$X_{p}(t)=\int_{0}^{t} \Lambda_{p}(p \tau) X_{p}(\tau)^{t} d \tau$. Hence $\left\|X_{p}\right\| \leq C\|p\|\left\|X_{p}\right\|$ for some constant $C>0$ independent of $p$. It thus follows that $X_{p} \equiv 0$ once $\|p\|<\frac{1}{C}$. Since $\phi(0)=0$, this also gives that $\phi \equiv 0$ over $M_{1}$.

Once we showed that $\phi \equiv 0$, then $(f, g)$ is a $C^{2}$ CR diffeomorphism between two small open pieces (near the origin) of $\partial \mathbf{H}_{n}$. Applying a classical theorem of Alexander, we see that $(f, g)$ extends to an automorphism of $\mathbf{H}_{n}$. Since $(f, g)$ satisfies the normalization condition in (3.1), we get that $(f, g)=(z, w)$, say, by Lemma 3.0. (Here, after knowing $\phi \equiv 0$, we remark that we can also directly get $(f, g)=(z, w)$ by studying the uniqueness of the first $n-1$ components of Equation (4.1'), coupled with $L_{j}\left(L_{k}(g)\right)=2 i L_{j}\left(L_{k}(f)\right) \cdot \bar{f}$.) The proof of Theorem 4.2 is complete. q.e.d.

\section{Completion of the proof of Theorem A}

We now proceed to the proof of Theorem A. We start with the following:

Proposition 5.1. Let $F=(f, \phi, g)$ be any twice differentiable $C R$ mapping from $M_{1}$ into $M_{2}$ satisfying the normalization condition (3.1). Assume that $N \leq 2 n-2$. Then $f=z+o_{w t}(3), g=w+o_{w t}(4)$ and $\phi=o_{w t}(2)$.

In what follows, to simplify the notation, we use $P_{1}$ and $P_{2}$ for polynomials which may be different in different contexts. We say that $w t\left(P_{1}\right) \geq k$ if $\left|P_{1}\left(t z, t^{2} w, t \bar{z}, t^{2} \bar{w}\right)\right| \leq \mathrm{C} t^{k}$.

We proceed by presenting the following Lemma 5.2 and Lemma 5.3. Since Lemma 5.2 is elementary, we leave its proof to the reader. (Different from Proposition 5.1, the following two lemmas hold in any codimensional case.)

Lemma 5.2. (a) For any function $\chi \in C^{k}\left(M_{1}\right) \cap o_{w t}(k)$ with $k \geq 1$, $L_{j} \chi, \overline{L_{j}} \chi, \in o_{w t}(k-1)$, and $T \chi \in o_{w t}(k-2)$.

(b) If $\chi \in C^{1}\left(M_{1}\right)$ is such that $\overline{L_{j}}(\chi), L_{j}(\chi) \in \mathcal{P}+o_{w t}(k-1)$ for each $j$ and $T(\chi) \in \mathcal{P}+o_{w t}(k-2)$, then $\chi \in \mathcal{P}+o_{w t}(k)$. Moreover, write $\chi=h_{1}+h_{2}$, where $h_{1}$ is a polynomial with weighted degree not 
larger than $k$ and $h_{2} \in o_{w t}(k)$. Then in case $\chi$ is $C R, h_{1}$ must be a holomorphic polynomial in $(z, w)$.

Lemma 5.3. Let $F=(f, \phi, g)=\left(f_{1}, \cdots, f_{n-1}, \phi_{1}, \cdots, \phi_{k}, g\right)$ be a twice differentiable $C R$ map from $M_{1}$ into $M_{2}$ satisfying the normalization condition (3.1). Then, $f=Q+o_{w t}(3)$ and $g=P+$ $o_{w t}(4)$ with $P, Q$ certain holomorphic polynomials. In fact, we can choose $P, Q$ such that $P=w$ and $Q=z+\frac{i w}{2} a^{(1)}(z)$, where $a^{(1)}(z)$ is a certain (vector-valued) holomorphic polynomial of degree one with $<a^{(1)}(z), \bar{z}>=\sum_{j=1}^{k}\left|\phi_{j}^{(2)}(z)\right|^{2}$.

Proof of Lemma 5.3. We first recall that

$$
\frac{1}{2 i} L_{l} g=\sum L_{l}\left(f_{j}\right) \overline{f_{j}}+\sum L_{l}\left(\phi_{j}\right) \overline{\phi_{j}}
$$

Also, it is easy to verify the following:

$$
L_{l} f_{j} \overline{f_{j}}=\left(P_{1}+o_{w t}(1)\right)\left(P_{2}+o_{w t}(2)\right) \in \mathcal{P}+o_{w t}(2),
$$

where $w t\left(P_{2}\right) \geq 1$; and

$$
L_{l} \phi_{j} \overline{\phi_{j}}=\left(P_{1}+o_{w t}(1)\right)\left(P_{2}+o_{w t}(2)\right) \in \mathcal{P}+o_{w t}(3),
$$

where $w t\left(P_{1}\right) \geq 1, w t\left(P_{2}\right) \geq 2$. Hence, $L_{l} g \in \mathcal{P}+o_{w t}(2)$.

On the other hand, since $g \in C^{2}\left(M_{1}\right), T(g) \in \mathcal{P}+o_{w t}(1)$. By Lemma 5.1 (b), we see that $g \in \mathcal{P}+o_{w t}(3)$.

Notice $g=w+o_{w t}(2)$. Write $\hat{g}=\hat{g}_{3}+o_{w t}(3)$ with $\hat{g}_{3}$ a weighted homogeneous polynomial of degree 3 . By Lemma $5.2(\mathrm{~b}), \hat{g}_{3}$ is holomorphic. To see that $\hat{g}^{(3)}=\hat{g}_{3}=0$ and $\hat{f}^{(2)}=0$, it now suffices for us to apply Lemma 3.0 to $\left(3.3^{\prime \prime}\right)$ with $k=1$ (this can also be quite easily seen by a straightforward computation).

Next, we apply $L_{j}$ to $(5.0)$ to yield $\frac{1}{2 i} L_{j}\left(L_{l} g\right)=L_{j}\left(L_{l}(\tilde{f})\right) \cdot \overline{\widetilde{f}}$. Applying the just obtained equation by $\overline{L_{k}}$ and making use of Lemma 4.1, one gets $L_{j}\left(L_{l}(\tilde{f})\right) \cdot \overline{L_{k} \widetilde{f}}=0$, for $j, l$ different from $k$, and

$$
L_{j}\left(L_{l}(\widetilde{f})\right) \cdot \overline{L_{k} \widetilde{f}}+c T\left(L_{v}(\widetilde{f})\right) \cdot \overline{\widetilde{f}}=\frac{c}{2 i} T L_{v}(g),
$$

for $k=j$ or $k=l$. Here when $l \neq j$, we have $c=2 i, v=\{l, j\} \backslash\{k\}$; and when $l=j$, we have $c=4 i, v=k$. Since $L_{k}\left(f_{\mu}\right)=\delta_{\mu}^{k}+o_{w t}(1)$, we can easily solve the above equations $L_{j} L_{l}\left(f_{k}\right)$ and then verify that

$$
L_{j} L_{l}\left(f_{k}\right)-\frac{c T L_{l}(g)}{2 i L_{k}\left(f_{k}\right)} \in \mathcal{P}+o_{w t}(1)
$$


Since

$$
2 i T\left(L_{l}(g)\right)=L_{l}(T(\widetilde{f})) \cdot \overline{\widetilde{f}}+\left(L_{l}(\widetilde{f})\right) \cdot T(\widetilde{f}),
$$

it follows readily that $T\left(L_{l}(g)\right) \in \mathcal{P}+o_{w t}(1)$. Therefore, we prove that $L_{j}\left(L_{l}\left(f_{k}\right)\right) \in \mathcal{P}+o_{w t}(1)$ for any $j, l, k$.

Since $F$ is assumed to be twice differentiable, $\overline{L_{j}}\left(L_{l} f_{k}\right)=\delta_{l}^{j} T\left(f_{k}\right) \in$ $\mathcal{P}+o_{w t}(1)$ and $T\left(L_{l}\left(f_{k}\right)\right) \in \mathcal{P}+o_{w t}(0)$. Making use of Lemma 5.2 , we see that $L_{l}\left(f_{k}\right) \in \mathcal{P}+o_{w t}(2)$ for any $l, k$. Again since $T\left(f_{k}\right) \in \mathcal{P}+o_{w t}(1)$, $f_{k} \in \mathcal{P}+o_{w t}(3)$ for any $k$. Also, by Lemma $5.2(\mathrm{~b})$, we can write $f=Q+o_{w t}(3)$ for a certain holomorphic polynomial $Q$. Making use of what we obtained above, it is now easy to verify that the expressions $L_{l} f_{j} \overline{f_{j}}$ and $\left\|L_{l} f_{j}\right\|^{2}$ stay in $\mathcal{P}+o_{w t}(3)$ and $\mathcal{P}+o_{w t}(2)$, respectively. By the identity (5.0), $2 i T(g)=<2 i T f, \bar{f}>+\left\|L_{l} f\right\|^{2}+<2 i T \phi, \bar{\phi}>+\left\|L_{l} \phi\right\|^{2}$, and the same argument as before, we can see that $L_{j}(g) \in \mathcal{P}+o_{w t}(3)$ and $T(g) \in \mathcal{P}+o_{w t}(2)$. Similarly, $g=P+o_{w t}(4)$ for a certain holomorphic polynomial $P$. Finally, applying the first part of Proposition 3.1 to $\left(3.3^{\prime}\right)$, we complete the proof of Lemma 5.3. q.e.d.

Proof of Proposition 5.1. By Lemma 5.3, we can write $f=z+\hat{f}^{(3)}+$ $o_{w t}(3)$ and $g=w+o_{w t}(4)$, where $f^{(3)}$ is a holomorphic polynomials of weighted degree 3 . To finish the proof of Proposition 5.1, it suffices to apply the last part of Proposition 3.1 to $\left(3.3^{\prime}\right)$. q.e.d.

Finally, we are ready to finish the proof of Theorem A:

Proof of Theorem A. Let $F$ be as in Theorem A and assume that $N \leq 2 n-2$. After the normalization in $\S 2$, we may assume that $F$ satisfies the normalization condition in (3.1). Now, as did in $\S 4$, using the Heisenberg group structures of $\partial \mathbf{H}_{n}$ and $\partial \mathbf{H}_{N}$, for any $\left(z_{0}, w_{0}\right) \approx 0$ and $\left(C_{l}\right)_{\left(z_{0}, w_{0}\right)}$ as chosen before, we have the new twice differentiable CR map $F_{\left(z_{0}, w_{0}\right)}^{* *}$ from $M_{1}$ into $M_{2}$, which still satisfies the normalization condition (3.1). Applying Proposition 5.1, we see that $f_{\left(z_{0}, w_{0}\right)}^{* *}$ $=z+o_{w t}(3), g_{\left(z_{0}, w_{0}\right)}^{* *}=w+o_{w t}(4)$ and $\phi_{\left(z_{0}, w_{0}\right)}^{* *}=o_{w t}(2)$ always hold. Hence, from Theorem 4.2 it follows that $F=(z, 0, w)$. This completes the proof of Theorem A. q.e.d.

\section{References}

[1] H. Alexander, Holomorphic mappings from ball and polydisc, Math. Ann. 209 (1974) 245-256. 
[2] _ Proper holomorphic maps in $\mathbf{C}^{n}$, Indiana Univ. Math. J. 26 (1977) $137-146$.

[3] M. S. Baouendi, H. Jacobowitz \& F. Treves, On the real analyticity of CR mappings, Ann. of Math. 122 (1985) 365-400.

[4] M. S. Baouendi \& L. P. Rothschild, Geometric properties of smooth and holomorphic mappings between surfaces in complex spaces, J. Differential Geom. 31 (1990) 473-499.

[5] S. Baouendi, X. Huang \& L. Rothschild, Regularity of CR mappings between algebraic hypersurfaces, Invent. Math. 125 (1996) 13-36.

[6] S. Bell \& R. Narasimhan, Proper holomorphic mappings of complex spaces, Encyclopedia Math. Sci. 69, Several Complex Variables VI (eds. W. Barth and R. Narasimhan), Springer, Berlin, 1990.

[7] D. Catlin \& J.P. D'Angelo, A stabilization theorem for Hermitian forms and applications to holomorphic mappings, Math. Res. Lett. 3 (1996) 149-166.

[8] S. S. Chern \& J. K. Moser, Real hypersurfaces in complex manifolds, Acta Math. 133 (1974) 219-271.

[9] J. Cima \& T. J. Suffridge, A reflection principle with applications to proper holomorphic mappings, Math. Ann. 265 (1983) 489-500.

[10] Boundary behavior of rational proper maps, Duke Math. J. 60 (1990) $135-138$

[11] J. Cima, S. Krantz \& T. J. Suffridge, A reflection principle for proper holomorphic mappings of strictly pseudoconvex domains and applications, Math. Z. 186 (1984) 1-8.

[12] H. Cao \& N. Mok, Holomorphic immersions between compact hyperbolic space forms, Invent. Math. 100 (1990) 49-61.

[13] J. P. D'Angelo, Several complex variables and the geometry of real hypersurfaces, CRC Press, Boca Raton, 1993.

[14] J. Faran, Maps from the two ball to the three ball, Invent. Math. 68 (1982) 441-475.

[15] _ On the linearity of proper maps between balls in the lower dimensional case, J. Differential Geom. 24 (1986) 15-17.

[16] _ A reflection principle for proper holomorphic mappings and geometric invariants, Math. Z. 203 (1990) 363-377.

[17] F. Forstneric, Proper holomorphic maps between balls, Duke Math. J. 53 (1986) $427-440$

[18] - Extending proper holomorphic mappings of positive codimension, Invent. Math. 95 (1989) 31-62 . 
[19] _ A survey on proper holomorphic mappings, Proc. Year in Several Complex Variables at Mittag-Leffler Institute, Math. Notes 38 (1992), Princeton University Press, Princeton, NJ.

[20] M. Hakim \& N. Sibony, Fonctions holomorphes bornes sur la boule unite de $\mathbf{C}^{n}$, Invent. Math. 67 (1982) 213-222.

[21] X. Huang, On the mapping problem for algebraic hypersurfaces in complex spaces of different dimensions, Ann. Inst. Fourier (Grenoble) 44 (1994) 433-463.

[22] L. Lempert, Embedding strictly pseudoconvex domains into the ball, Amer. J. Math. 104 (1982) 901-904.

[23] - Embedding Cauchy-Riemann manifolds into a sphere, Internat. J. Math. (1990) 91-100.

[24] E. Low, Embeddings and proper holomorphic mappings of strictly pseudoconvex domains into polydiscs and balls, Math. Z. 190 (1985) 401-410.

[25] J. Moser, Analytic surfaces in $\mathbf{C}^{2}$ and their local hull of holomorphy, Ann. Acad. Sci. Fenn. Ser. A I Math. 10 (1985) 397-410.

[26] H. Poincaré, Les fonctions analytiques de deux variables et la représentation conforme, Rend. Circ. Mat. Palermo, (2) 23 (1907) 185-220.

[27] B. Stensones, Proper holomorphic mappings between the balls, J. Geom. Anal., to appear, 1994.

[28] N. Tanaka, On the pseudo-conformal geometry of hypersurfaces of the space of $n$ complex variables, J. Math. Soc. Japan 14 (1962) 397-429.

[29] V. Vitushikin, Holomorphic mappings and the geometry of hypersurfaces, several complex variables. I, Encyclopedia Math. Sci., 7. Springer, Berlin, (1990) 159-215.

[30] S. Webster, On the mapping problem for algebraic real hypersurfaces, Invent. Math. 43 (1977) 53-68.

[31] On mappings an $(n+1)$-ball in the complex space, Pacific J. Math. 81 (1979) 267-272.

[32] C. Yue, Dimension and rigidity of quasi-Fuchsian representations, Ann. of Math. (2) 143 (1996) 331-355.

RUTGERS UNIVERSITY 